ELECTRONIC RESEARCH ANNOUNCEMENTS OF THE AMERICAN MATHEMATICAL SOCIETY

Volume 10, Pages 78-87 (August 3, 2004)

S $1079-6762(04) 00132-5$

\title{
WAVELETS WITH COMPOSITE DILATIONS
}

\author{
KANGHUI GUO, DEMETRIO LABATE, WANG-Q LIM, GUIDO WEISS, \\ AND EDWARD WILSON
}

(Communicated by Boris Hasselblatt)

\begin{abstract}
A wavelet with composite dilations is a function generating an orthonormal basis or a Parseval frame for $L^{2}\left(\mathbb{R}^{n}\right)$ under the action of lattice translations and dilations by products of elements drawn from non-commuting matrix sets $A$ and $B$. Typically, the members of $B$ are shear matrices (all eigenvalues are one), while the members of $A$ are matrices expanding or contracting on a proper subspace of $\mathbb{R}^{n}$. These wavelets are of interest in applications because of their tendency to produce "long, narrow" window functions well suited to edge detection. In this paper, we discuss the remarkable extent to which the theory of wavelets with composite dilations parallels the theory of classical wavelets, and present several examples of such systems.
\end{abstract}

\section{INTRODUCTION}

There is considerable interest, both in mathematics and its applications, in the study of efficient representations of multidimensional functions. The motivation comes partly from signal processing, including applications in image compression and feature extraction, and from the investigation of certain classes of singular integral operators. In particular, it was pointed out in several recent research papers that oriented oscillatory waveforms play a fundamental role in the construction of representations for multidimensional functions and signals (cf. [1], 3], [5], and articles in 11]). For example, it was shown that, in order to be optimally sparse in a certain sense, such representations must contain basis elements with many locations, scales, shapes and directions, unlike the trigonometric bases or even the "classical" wavelets, which are made of essentially isotropic oscillatory bumps at various scales and locations (cf. [2]).

In this paper, we introduce a new class of representation systems which have exactly the features we have described, as well as several other properties which have a great potential in applications. These systems, that we call affine systems with composite dilations, have the form

$$
\Psi_{A B}=\left\{D_{a} D_{b} T_{k} \psi^{\ell}: k \in \mathbb{Z}^{n}, b \in B, a \in A, \ell=1, \ldots, L\right\},
$$

Received by the editors February 23, 2004 and, in revised form, April 13, 2004

2000 Mathematics Subject Classification. Primary 42C15, 42C40.

Key words and phrases. Affine systems, frames, multiresolution analysis (MRA), multiwavelets, wavelets.

The fourth author was supported in part by a SW Bell Grant.

(C)2004 American Mathematical Society 
where $\psi^{\ell} \in L^{2}\left(\mathbb{R}^{n}\right), T_{k}$ are the translations defined by $T_{k} f(x)=f(x-k), D_{a}$ are the dilations defined by $D_{a} f(x)=|\operatorname{det} a|^{-1 / 2} f\left(a^{-1} x\right)$, and $A, B$ are countable subsets of $G L_{n}(\mathbb{R})$. By choosing $\psi^{\ell}, A$, and $B$ appropriately, we can make $\Psi_{A B}$ an orthonormal (ON) basis or, more generally, a Parseval frame $(\mathrm{PF})$ for $L^{2}\left(\mathbb{R}^{n}\right)$. In this case, we call $\Psi=\left\{\psi^{1}, \ldots, \psi^{L}\right\}$ an $\mathbf{O N} A B$-multiwavelet or a PF $A B$ multiwavelet, respectively. If the system has only one generator, that is, $L=1$, then we use the expression wavelet rather than multiwavelet in this definition.

As we will show, the mathematical theory of these systems provides a simple and flexible framework for the construction of several classes of bases and Parseval frames. For example, in Sections 3.3 and 3.4 we construct composite wavelets with good time-frequency decay properties, whose elements contain "long and narrow" waveforms with many locations, scales, shapes and directions. These examples have similarities to the curvelets [1] and contourlets [4], which have been recently introduced in order to obtain efficient representations of natural images. Our approach is more general and presents a simple method for obtaining orthonormal bases and Parseval frames that exhibit these and other geometric features. In particular, our approach extends naturally to higher dimensions and allows a multiresolution construction which is well suited to a fast numerical implementation.

Before embarking on our presentation, it is useful to establish some notation and definitions. Recall that a countable family $\left\{\psi_{i}\right\}_{i \in \mathcal{I}}$ of elements in a separable Hilbert space $\mathcal{H}$ is a Parseval frame (PF) for $\mathcal{H}$, if

$$
\|f\|^{2}=\sum_{i \in \mathcal{I}}\left|\left\langle f, \psi_{i}\right\rangle\right|^{2}
$$

for each $f \in \mathcal{H}$. We adopt the convention that an element $x \in \mathbb{R}^{n}$ is a column vector, while $\xi \in \widehat{\mathbb{R}}^{n}$ is a row vector. A vector $x$ multiplying a matrix $a \in G L_{n}(\mathbb{R})$ on the right, is understood to be a column vector, while a vector $\xi$ multiplying $a$ on the left is a row vector. Thus, $a x \in \mathbb{R}^{n}$ and $\xi a \in \widehat{\mathbb{R}}^{n}$. The Fourier transform is defined as

$$
\hat{f}(\xi)=\int_{\mathbb{R}^{n}} f(x) e^{-2 \pi i \xi x} d x
$$

For any $E \subset \widehat{\mathbb{R}}^{n}$, we denote by $L^{2}(E)^{\vee}$ the space $\left\{f \in L^{2}\left(\mathbb{R}^{n}\right): \operatorname{supp} \hat{f} \subset E\right\}$.

\section{2. $A B$-MRA}

Let $B$ be a countable subset of $\widetilde{S L_{n}}(\mathbb{Z})=\left\{b \in G L_{n}(\mathbb{R}):|\operatorname{det} b|=1\right\}$ and $A=$ $\left\{a_{i}: i \in \mathbb{Z}\right\}$, where $a_{i} \in G L_{n}(\mathbb{R})$. We say that a sequence $\left\{V_{i}\right\}$ of closed subspaces of $L^{2}\left(\mathbb{R}^{n}\right)$ is an $A B$-multiresolution analysis ( $A B$-MRA) if the following holds:

(i) $D_{b} T_{k} V_{0}=V_{0}$, for any $b \in B, k \in \mathbb{Z}^{n}$.

(ii) For each $i \in \mathbb{Z}, V_{i} \subset V_{i+1}$,, where $V_{i}=D_{a_{i}}^{-1} V_{0}$.

(iii) $\bigcap V_{i}=\{0\}$ and $\overline{U V_{i}}=L^{2}\left(\mathbb{R}^{n}\right)$.

(iv) There exists $\phi \in L^{2}\left(\mathbb{R}^{n}\right)$ such that $\Phi_{B}=\left\{D_{b} T_{k} \phi: b \in B, k \in \mathbb{Z}^{n}\right\}$ is a semi-orthogonal $P F$ for $V_{0}$, that is, $\Phi_{B}$ is a $P F$ for $V_{0}$ and, in addition, $D_{b} T_{k} \phi \perp D_{b^{\prime}} T_{k^{\prime}} \phi$ for any $b \neq b^{\prime}, b, b^{\prime} \in B, k, k^{\prime} \in \mathbb{Z}^{n}$.

The space $V_{0}$ is called an $A B$ scaling space and the function $\phi$ is an $A B$ scaling function for $V_{0}$. If, in addition, $\Phi_{B}$ is an orthonormal basis for $V_{0}$, then $\phi$ is an ON $A B$ scaling function. Also, let $W_{0}$ be the orthogonal complement of $V_{0}$ in $V_{1}$, that is, $V_{1}=V_{0} \oplus W_{0}$. We cite the following elementary result [6]: 
Theorem 2.1. $\quad$ (i) Let $\Psi=\left\{\psi^{1}, \ldots, \psi^{L}\right\} \subset L^{2}\left(\mathbb{R}^{n}\right)$ be such that $\left\{D_{b} T_{k} \psi^{\ell}\right.$ : $\left.b \in B, \ell=1, \ldots, L, k \in \mathbb{Z}^{n}\right\}$ is a $P F$ for $W_{0}$. Then $\Psi$ is a $P F A B$ multiwavelet.

(ii) Let $\Psi=\left\{\psi^{1}, \ldots, \psi^{L}\right\} \subset L^{2}\left(\mathbb{R}^{n}\right)$ be such that $\left\{D_{b} T_{k} \psi^{\ell}: b \in B, k \in\right.$ $\left.\mathbb{Z}^{n}, \ell=1, \ldots, L\right\}$ is an orthonormal basis for $W_{0}$. Then $\Psi$ is an $O N A B$ multiwavelet.

In the situation described by the hypotheses of this theorem (where $\Psi$ is not only a PF for $L^{2}\left(\mathbb{R}^{n}\right)$, but it is also derived from an $A B$-MRA), we say that $\Psi$ is a PF MRA $A B$-multiwavelet or an ON MRA $A B$-multiwavelet, respectively.

In most cases of interest, the set $A \subset G L_{n}(\mathbb{R})$ has the form $A=\left\{a_{i}=a^{i}: i \in \mathbb{Z}\right\}$, where $a \in G L_{n}(\mathbb{R})$, and $B$ is a subgroup of $\widetilde{S L}_{n}(\mathbb{Z})$ which satisfies $a B a^{-1} \subseteq B$. For the remainder of this section, we will make this assumption.

We say that the PF MRA $A B$-wavelet $\psi$ is of finite filter (FF) type if there exists an $A B$ scaling function $\phi$ for $V_{0}$ and a finite set $\left\{b_{1}, \ldots, b_{k}\right\} \subset B$ such that

$$
\hat{\phi}(\xi a)=\sum_{j=1}^{k} m_{0}^{(j)}(\xi) \hat{\phi}\left(\xi b_{j}\right), \quad \hat{\psi}(\xi a)=\sum_{j=1}^{k} m_{1}^{(j)}(\xi) \hat{\phi}\left(\xi b_{j}\right),
$$

where $m_{0}^{(j)}, m_{1}^{(j)}, 1 \leq j \leq k$, are periodic functions. Similarly, the ON MRA $A B$ multiwavelet $\Psi$ is of finite filter (FF) type if there exists an $A B$ scaling function $\phi$ for $V_{0}$ and a finite set $\left\{b_{1}, \ldots, b_{k}\right\} \subset B$ such that

$$
\hat{\phi}(\xi a)=\sum_{j=1}^{k} m_{0}^{(j)}(\xi) \hat{\phi}\left(\xi b_{j}\right), \quad \hat{\psi}^{\ell}(\xi a)=\sum_{j=1}^{k} m_{1, \ell}^{(j)}(\xi) \hat{\phi}\left(\xi b_{j}\right), \ell=1, \ldots, L,
$$

where $m_{0}^{(j)}, m_{1, \ell}^{(j)}, 1 \leq j \leq k$, are periodic functions.

It turns out that, while it is possible to construct a $\mathrm{PF} A B$-wavelet using a single generator, that is, $\Psi=\{\psi\}$, in the case of orthonormal MRA $A B$-multiwavelets, multiple generators are needed, that is, $\Psi=\left\{\psi^{1}, \ldots, \psi^{L}\right\}$, where $L>1$. This situation is similar to the classical MRA and, as in that case, such restriction is not needed if the system does not come from an MRA (cf., for example, [12]). We refer to [6] for a proof of the following theorems.

Theorem 2.2. Let $\Psi=\left\{\psi^{1}, \ldots, \psi^{L}\right\}$ be an ON MRA AB-multiwavelet for $L^{2}\left(\mathbb{R}^{n}\right)$, and let $N=\left|B / a B a^{-1}\right|$ (= the order of the quotient group $\left.B / a B a^{-1}\right)$. Assume that $|\operatorname{det} a| \in \mathbb{N}$. Then $L=N|\operatorname{det} a|-1$.

Theorem 2.3. Let $B \subset \widetilde{S L}_{n}(\mathbb{Z})$ and $a \in G L_{n}(\mathbb{Z})$ with $a B a^{-1} \subseteq B$. Let $L=$ $N|\operatorname{det} a|-1$, where $N=\left|B / a B a^{-1}\right|$. Assume that $\phi \in L^{2}\left(\mathbb{R}^{n}\right)$ is an $O N A B$ scaling function for $V_{0}=\overline{\operatorname{span}\left\{D_{b} T_{k} \phi: k \in \mathbb{Z}^{n}, b \in B\right\}}$. Then:

(i) There exist ON MRA AB-multiwavelets $\Psi=\left\{\psi^{1}, \ldots, \psi^{L}\right\}$ with scaling space $V_{0}$.

(ii) If $\hat{\phi}=\chi_{U}$, where $U \subset \mathbb{R}^{n}$ is a measurable set, then there are sets $T_{\ell} \subset \mathbb{R}^{n}$, $\ell=1, \ldots, L$, for which $\Psi=\left\{\psi^{\ell}=\left(\chi_{T_{\ell}}\right)^{\vee}: \ell=1, \ldots, L\right\}$ is an $O N M R A$ $A B$-multiwavelet and $\Psi$ is of FF type.

Remark 2.4. Let $a \in G L_{n}(\mathbb{Z})$. Under additional assumptions on $B$ (which are satisfied, for example, by the examples in Section [3), there exist sets $S \subset \widehat{\mathbb{R}}^{n}$ such that $\psi$ is an ON $A B$-wavelet for $L^{2}\left(\mathbb{R}^{n}\right)$, where $\hat{\psi}=\chi_{S}$. It is clear, by Theorem 2.2 that these $A B$-wavelets are not of MRA type. 


\section{EXAMPLES}

This section shows that there are several examples of affine systems with composite dilations forming ON bases or PFs for $L^{2}\left(\mathbb{R}^{n}\right)$. In particular, Sections 3.1 and 3.2 contain examples of PF MRA $A B$-multiwavelets and ON MRA $A B$-multiwavelets for $L^{2}\left(\mathbb{R}^{2}\right)$, respectively; Sections 3.3 and 3.4 describe how to construct PF $A B$ wavelets which are well-localized both in $\mathbb{R}^{2}$ and $\widehat{\mathbb{R}}^{2}$; Section 3.5 describes generalizations of these examples for dimensions $n>2$; finally, Section 3.6 describes a class of singly generated (non-MRA) ON $A B$-wavelets. More details about these constructions are found in 6 .

3.1. Example 1. Let $B=\left\{b_{j}=\left(\begin{array}{ll}1 & j \\ 0 & 1\end{array}\right): j \in \mathbb{Z}\right\}$. For $0 \leq \alpha<\beta$, let $S(\alpha, \beta)=$ $\left\{\xi=\left(\xi_{1}, \xi_{2}\right) \in \widehat{\mathbb{R}}^{2}: \alpha \leq\left|\xi_{1}\right|<\beta\right\}$ and let $T(\alpha, \beta)=T^{+}(\alpha, \beta) \cup T^{-}(\alpha, \beta)$, where $T^{+}(\alpha, \beta)$ is the trapezoid with vertices $(\alpha, 0),(\alpha, \alpha),(0, \beta)$ and $(\beta, \beta)$, and $T^{-}(\alpha, \beta)=\left\{\xi \in \widehat{\mathbb{R}}^{2}:-\xi \in T^{+}(\alpha, \beta)\right\}$. Observe that $T(0, \beta)$ is the union of two triangles. A simple computation shows that $T(\alpha, \beta)$ is a $B$-tiling region for $S(\alpha, \beta)$, that is, $S(\alpha, \beta)$ is the disjoint union, modulo null sets, of the sets $T(\alpha, \beta) b$, $b \in B$.

Now let $a=\left(\begin{array}{cc}c & d \\ 0 & e\end{array}\right) \in G L_{2}(\mathbb{R})$, with $|c|>1, S_{0}=S(0,1 / 2|c|), S_{i}=S_{0} a^{i}, i \in \mathbb{Z}$, and define a sequence $\left\{V_{i}\right\}_{i \in \mathbb{Z}}$ of nested subspaces of $L^{2}\left(\mathbb{R}^{2}\right)$ by $V_{i}=L^{2}\left(S_{i}\right)^{\vee}, i \in \mathbb{Z}$. Also, let $U=T\left(0,(2|c|)^{-1}\right)$ and $\phi=\left(\chi_{U}\right)^{\vee}$. Since $U$ is a $B$-tiling region for $S_{0}$, it follows that $\overline{\operatorname{span}\left\{D_{b} T_{k} \phi: k \in \mathbb{Z}^{2}, b \in B\right\}}=L^{2}\left(S_{0}\right)^{\vee}=V_{0}$. This shows that the spaces $\left\{V_{i}\right\}$ form an $A B$-MRA and $\phi$ is the $A B$ scaling function for $V_{0}$. In addition, for $R=T\left((2|c|)^{-1}, 2^{-1}\right)$, the function $\psi=\left(\chi_{R}\right)^{\vee}$ is a PF MRA $A B$-wavelet, since $\left\{D_{b} T_{k} \psi: b \in B, k \in \mathbb{Z}^{2}\right\}$ is a $\mathrm{PF}$ for $W_{0}=L^{2}\left(S_{1} \backslash S_{0}\right)^{\vee}$. Observe that $W_{0}$ is the orthogonal complement of $V_{0}$ in $V_{1}$. Also, $\psi$ is of FF type, since $U a^{-1}$ and $R a^{-1}$ are contained in $\bigcup_{j=0}^{k-1} U b_{j}$ for $k \geq|c|$.

3.2. Example 2. Let $B, S(\alpha, \beta)$, and $T(\alpha, \beta)$ be defined as in Example 1. Let $a=\left(\begin{array}{cc}k_{1} & k_{2} \\ 0 & k_{3}\end{array}\right) \in G L_{2}(\mathbb{Z})$, with $k_{1}>1$ and $N=\left|k_{1} / k_{3}\right| \in \mathbb{N}$, and let $L=|\operatorname{det} a| N-1$ $=k_{1}^{2}-1$. Similarly to Example 1 , let $S_{0}=S(0,1), S_{i}=S_{0} a^{i}, i \in \mathbb{Z}$, and define a sequence of spaces $\left\{V_{i}\right\}_{i \in \mathbb{Z}}$ by $V_{i}=L^{2}\left(S_{i}\right)^{\vee}, i \in \mathbb{Z}$. Let $U=T(0,1)$ and $\phi=\left(\chi_{U}\right)^{\vee}$. Then $\phi$ is an ON basis for $\overline{\operatorname{span}\left\{D_{b} T_{k} \phi: k \in \mathbb{Z}^{n}, b \in B\right\}}=L^{2}\left(S_{0}\right)^{\vee}=V_{0}$, and the spaces $\left\{V_{i}\right\}_{i \in \mathbb{Z}}$ form an $A B$-MRA, where $\phi$ is the corresponding ON $A B$ scaling function.

Observe that the set $R=T\left(1, k_{1}\right)$ is a $B$-tiling region for $S_{1} \backslash S_{0}$. We can pick disjoint subsets $R_{\ell}, \ell=1, \ldots, L$ of $R$ such that $R=\bigcup_{\ell=1}^{L} R_{\ell}$ and, for each $\ell$, $R_{\ell}$ is a fundamental domain for $\mathbb{Z}^{2}$. Figure 1 illustrates this construction in the special case where $k_{1}=1$ and, thus, $L=3$. Now let $\Psi=\left\{\psi^{1}, \ldots, \psi^{L}\right\}$, where $\psi^{\ell}=\left(\chi_{R_{\ell}}\right)^{\vee}, \ell=1, \ldots, L$. Since $\left\{D_{b} T_{k} \psi^{\ell}: b \in B, \ell=1, \ldots, L, k \in \mathbb{Z}^{2}\right\}$ is an ON basis for $W_{0}=L^{2}\left(S_{1} \backslash S_{0}\right)^{\vee}$, and $W_{0}$ is the orthogonal complement of $V_{0}$ in $V_{1}$, it follows that $\Psi$ is an ON MRA $A B$-multiwavelet. In addition, $\Psi$ is of $\mathrm{FF}$ type, since the sets $U a^{-1}$ and $R_{\ell} a^{-1}, \ell=1, \ldots, L$, are contained in $\bigcup_{j=0}^{k_{1}-1} U b_{j}$.

The examples described in Sections 3.1 and 3.2 are well-localized in $\widehat{\mathbb{R}}^{n}$, since the $A B$-multiwavelets that we constructed are band-limited, but they do not have good localization in $\mathbb{R}^{n}$, since they decay only as fast as $|x|^{-1}$ when $|x| \rightarrow \infty$. The examples described in the next two sections, on the contrary, are well-localized both in $\mathbb{R}^{n}$ and $\widehat{\mathbb{R}}^{n}$. 


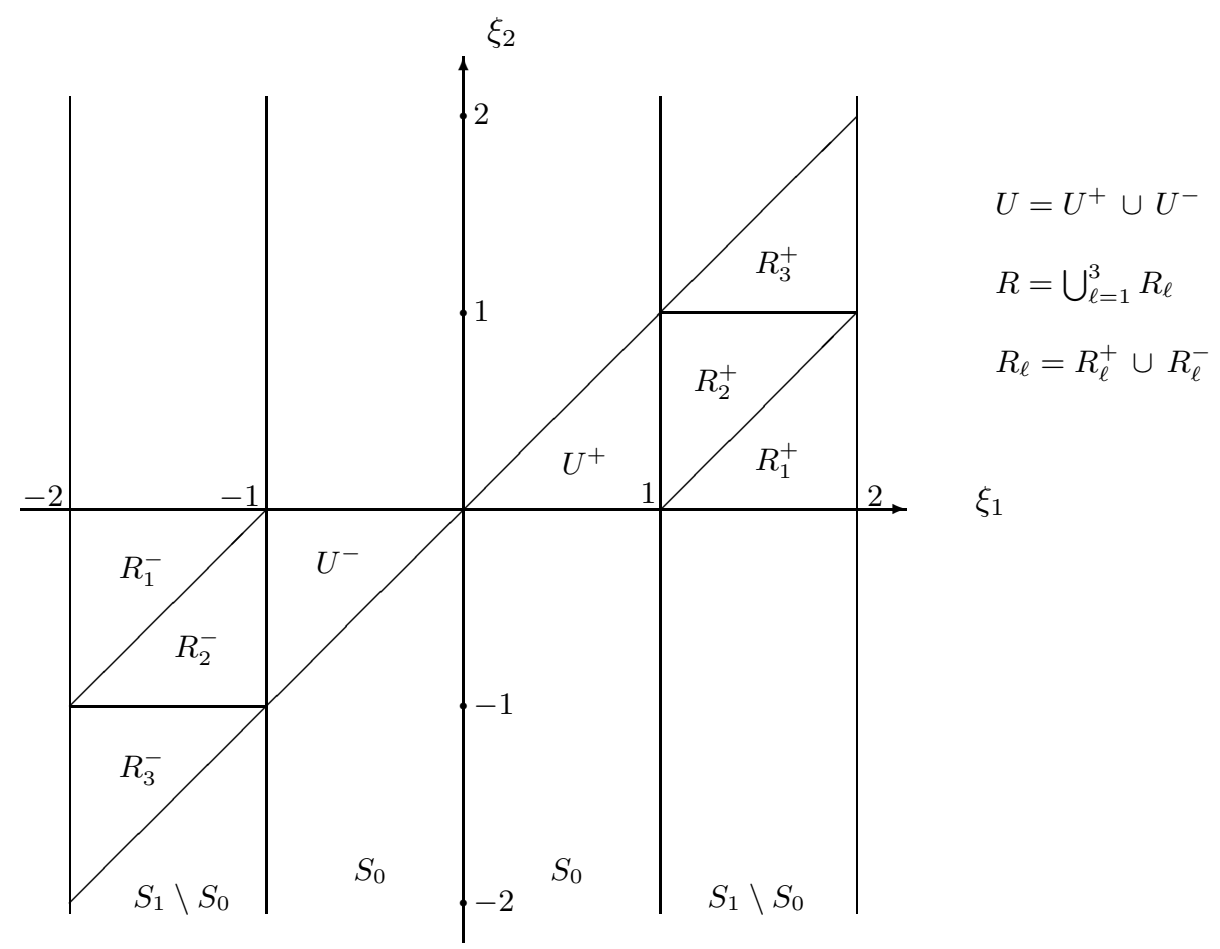

Figure 1. The sets $U, R$ and $R_{\ell}, \ell=1,2,3$, in Example 2, where $L=3$.

3.3. Example 3. Let $\psi_{1} \in L^{2}(\mathbb{R})$ be a (one-dimensional) dyadic band-limited wavelet with supp $\hat{\psi}_{1} \subset[-\Omega, \Omega], \Omega>0$, and $\psi_{2} \in L^{2}(\mathbb{R})$ be another band-limited function with supp $\hat{\psi}_{2} \subset[-1,1]$ and satisfying

$$
\sum_{j \in \mathbb{Z}}\left|\hat{\psi}_{2}(\xi+j)\right|^{2}=1 \quad \text { a.e. } \xi \in \mathbb{R} .
$$

As we will show later on, there are several choices of functions $\psi_{1}$ and $\psi_{2}$ satisfying these properties.

For any $\omega=\left(\omega_{1}, \omega_{2}\right) \in \mathbb{R}^{2}, \omega_{1} \neq 0$, define $\psi \in L^{2}\left(\mathbb{R}^{2}\right)$ by

$$
\hat{\psi}(\omega)=\hat{\psi}_{1}\left(2^{s} \omega_{1}\right) \hat{\psi}_{2}\left(\frac{\omega_{2}}{\omega_{1}}\right) \text {, }
$$

where $s \in \mathbb{Z}$ satisfies $2^{s} \geq 2 \Omega$. It turns out that $\psi$ is a PF $A B$-wavelet, where $A=\left\{a_{i}=\left(\begin{array}{ll}2^{i} & 0 \\ 0 & 1\end{array}\right): i \in \mathbb{Z}\right\}$ and $B=\left\{b_{j}=\left(\begin{array}{ll}1 & j \\ 0 & 1\end{array}\right): j \in \mathbb{Z}\right\}$. The proof of this fact is based on an application of a result from [7, where the characterization equations for a very general class of Parseval frames are obtained.

As we mentioned before, there are many choices for the functions $\psi_{1}$ and $\psi_{2}$ that satisfy the assumptions we have described above. For example, we can choose $\psi_{1}$ to be the Lemariè-Meyer wavelet (see [8, Sec. 1.4]) defined by $\hat{\psi}_{1}(\xi)=e^{i \pi \xi} b(\xi)$, where

$$
b(\xi)=\left\{\begin{array}{lc}
\sin \left(\frac{\pi}{2}(3|\xi|-1)\right), & \frac{1}{3} \leq|\xi| \leq \frac{2}{3} \\
\sin \left(\frac{3 \pi}{4}\left(\frac{4}{3}-|\xi|\right)\right), & \frac{2}{3}<|\xi| \leq \frac{4}{3} \\
0 & \text { otherwise. }
\end{array}\right.
$$


In order to construct $\psi_{2}$, let $\phi$ be a compactly supported $C^{\infty}$ bump function, with $\operatorname{supp} \phi \subset[-1,1]$ (examples can be found in [10, Sec. 3.3] or [9, Sec. 1.4]), and define $\psi_{2}$ by

$$
\hat{\psi}_{2}(\xi)=\frac{\phi(\xi)}{\sqrt{\sum_{k \in \mathbb{Z}}|\phi(\xi+k)|^{2}}} .
$$

It is clear that $\psi_{2} \in C^{\infty}(\mathbb{R})$ and satisfies (3.1). It follows that $\hat{\psi}$, given by (3.2), is in $C^{\infty}\left(\mathbb{R}^{2}\right)$, and this implies that $|\psi(x)| \leq K_{N}(1+|x|)^{-N}, K_{N}>0$, for any $N \in \mathbb{N}$.

The following example shows how to construct MRA $A B$-wavelets for $L^{2}\left(\mathbb{R}^{2}\right)$ which are well-localized both in $\mathbb{R}^{n}$ and $\widehat{\mathbb{R}}^{n}$.

3.4. Example 4. Let $\psi_{1} \in L^{2}(\mathbb{R})$ be a (one-dimensional) dyadic band-limited MRA wavelet with supp $\hat{\psi}_{1} \subset[-\Omega, \Omega], \Omega>0$, and $\phi_{1}$ be its associated scaling function. Let $m_{0}$ and $m_{1}$ be the low pass and high pass filters, respectively, associated with $\phi_{1}$ and $\psi_{1}$, that is, $m_{0}, m_{1}$ are the periodic functions satisfying the equations

$$
\hat{\phi}_{1}\left(\omega_{1}\right)=m_{0}\left(\frac{\omega_{1}}{2}\right) \hat{\phi}_{1}\left(\frac{\omega_{1}}{2}\right) \quad \text { and } \quad \hat{\psi}_{1}\left(\omega_{1}\right)=m_{1}\left(\frac{\omega_{1}}{2}\right) \hat{\phi}_{1}\left(\frac{\omega_{1}}{2}\right) .
$$

Let $\psi_{2} \in L^{2}(\mathbb{R})$ be defined by

$$
\psi_{2}(x)=e^{i(N+1) \pi x}\left(\frac{\sin \pi x}{\pi x}\right)^{N+1},
$$

where $N \in \mathbb{N}$. That is, $\hat{\psi}_{2}$ is a basic spline of order $N$ (cf. [8]).

For $\omega=\left(\omega_{1}, \omega_{2}\right) \in \mathbb{R}^{2}, \omega_{1} \neq 0$, let $\phi \in L^{2}\left(\mathbb{R}^{2}\right)$ be defined by

$$
\hat{\phi}(\omega)=\hat{\phi}_{1}\left(2^{s} \omega_{1}\right) \frac{\hat{\psi}_{2}\left(\frac{\omega_{2}}{\omega_{1}}\right)}{\sqrt{\sum_{m \in \mathbb{Z}}\left|\hat{\psi}_{2}\left(\frac{\omega_{2}}{\omega_{1}}+m\right)\right|^{2}}},
$$

where $s \in \mathbb{Z}$ satisfies $2^{s} \geq 4 \Omega\left(\frac{N}{2}+1\right)$. This assumption on $s$ ensures that

$$
\operatorname{supp}\left\{\hat{\phi}_{1}\left(2^{s} \omega_{1}\right) \hat{\psi}_{2}\left(\frac{\omega_{2}}{\omega_{1}}\right)\right\} \subset\left[-\frac{1}{4}, \frac{1}{4}\right]^{2} .
$$

Also, let $\psi \in L^{2}\left(\mathbb{R}^{2}\right)$ be defined by

$$
\hat{\psi}(\omega)=\sum_{k=0}^{N+1} d_{k}^{(N)} m_{1}\left(2^{s-1} \omega_{1}\right) M_{0}\left(a^{-1} \omega\right) \hat{\phi}\left(\left(b^{T}\right)^{-k} a^{-1} \omega\right),
$$

where the matrices $a$ and $b$ are as in Section 3.3, $d_{k}^{(N)}=2^{-N}\left(\begin{array}{c}N+1 \\ k\end{array}\right)$, and $M_{0}(\omega)$ is the $\mathbb{Z}^{2}$-periodic function which, restricted to the fundamental region $\left[-\frac{1}{2}, \frac{1}{2}\right]^{2}$, is given by

$$
M_{0}(\omega)=\left(\frac{\sum_{m \in \mathbb{Z}}\left|\hat{\psi}_{2}\left(\frac{\omega_{2}}{\omega_{1}}+m\right)\right|^{2}}{\sum_{m \in \mathbb{Z}}\left|\hat{\psi}_{2}\left(2^{-1} \frac{\omega_{2}}{\omega_{1}}+m\right)\right|^{2}}\right)^{1 / 2}, \quad \omega \in\left[-\frac{1}{2}, \frac{1}{2}\right]^{2} .
$$

It turns out that $\psi$ is a PF MRA $A B$-wavelet for $L^{2}\left(\mathbb{R}^{2}\right)$. Indeed, the spaces $\left\{V_{j}\right.$ : $j \in \mathbb{Z}\}$, where $V_{j}=D_{a}^{-j} V_{0}, j \in \mathbb{Z}$, and $V_{0}=\overline{\operatorname{span}\left\{D_{b} T_{m} \phi: b \in B, m \in \mathbb{Z}^{2}\right\}}$, form an $A B$-MRA. Observe, however, that this system is somewhat different from those described in Section 2, since the spaces $V_{0}$ and $W_{0}=\overline{\operatorname{span}\left\{D_{b} T_{m} \psi: b \in B, m \in \mathbb{Z}^{2}\right\}}$ are not mutually orthogonal. Also, by construction, $\hat{\psi} \in C^{N}\left(\widehat{\mathbb{R}}^{2}\right)$, so $|\psi(x)| \leq$ $K_{N}(1+|x|)^{1-N}$, for some $K_{N}>0$. 
3.5. Extensions for $n>2$. For $n>2$, there are several generalizations of the examples described in the previous sections. The general idea is to write $\widehat{R}^{n}=F \oplus E$ and to define matrices $a, b$ such that:

(i) $E a \subseteq E$;

(ii) the induced action of $a$ on $\widehat{R}^{n} / E$ is expanding;

(iii) $b$ is the identity map on $E$ and $b-I$ maps $F$ to $E$.

This allows us to construct a space $V_{0}=L^{2}(S)^{\vee}$ as we did in the 2-dimensional examples, for strip domains $S=c \times E$, where $c \subset F$ is contained in a neighborhood of the origin.

In particular, in order to extend Examples 1 and 2, let $n=k+\ell$, and $A=\left\{a^{i}\right.$ : $i \in \mathbb{Z}\}$, where $a=\left(\begin{array}{cc}a_{0} & a_{1} \\ 0 & a_{2}\end{array}\right) \in G L_{n}(\mathbb{R})$, and $a_{0} \in G L_{k}(\mathbb{R})$ is an expanding matrix. The matrices $B$ can be chosen according to one of the following patterns.

(i) Let $k=1, \ell=n-1$ and $B=\left\{b_{j}=\left(\begin{array}{cc}1 & j \\ 0 & I_{\ell}\end{array}\right): j \in \mathbb{Z}^{\ell}\right\}$. Then $\psi=\left(\chi_{R}\right)^{\vee}$, where $R=\left\{\left(\xi_{0}, \ldots, \xi_{\ell}\right) \in \mathbb{R}^{n}: \frac{1}{2\left|a_{0}\right|} \leq\left|\xi_{0}\right|<\frac{1}{2}, 0 \leq \xi_{i} / \xi_{0}<1\right.$, for $1 \leq$ $i \leq \ell\}$, is a PF MRA $A B$-wavelet.

(ii) Let $k \geq \ell>0$ and

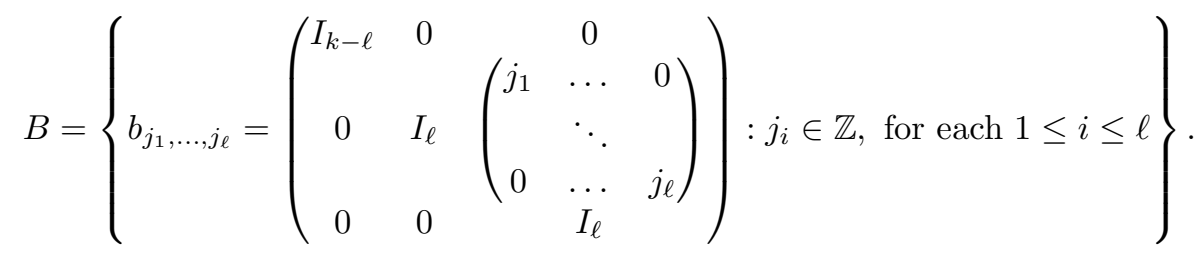

To illustrate this example, let $k=3, \ell=2$, and $a_{0}$ be a diagonal matrix with diagonal entries $c_{0}, c_{1}, c_{2}$ and $\left|c_{i}\right|>1, i=0,1,2$. Then $\psi=\left(\chi_{R}\right)^{\vee}$, where $R=\left\{\left(\xi_{0}, \ldots, \xi_{4}\right) \in \mathbb{R}^{n}: \frac{1}{2\left|c_{i}\right|} \leq\left|\xi_{i}\right|<\frac{1}{2}, i=0,1,2\right.$, and $0 \leq \xi_{i+2} / \xi_{i}<$ 1 , for $i=1,2\}$, is a PF MRA $A B$ wavelet. The construction is similar for any $k \geq \ell$.

(iii) Let $k=1, \ell=n-1$ and

$$
B=\left\{b_{j_{1}, \ldots, j_{\ell}}=\left(\begin{array}{ccccc}
1 & j_{1} & \cdots & 0 & 0 \\
0 & 1 & \ddots & 0 & 0 \\
& \ddots & \ddots & \ddots & \\
0 & 0 & \cdots & 1 & j_{\ell} \\
0 & 0 & \cdots & 0 & 1
\end{array}\right): j_{i} \in \mathbb{Z}, 1 \leq i \leq \ell\right\} .
$$

Then $\psi=\left(\chi_{R}\right)^{\vee}$, where $R=\left\{\left(\xi_{0}, \ldots, \xi_{\ell}\right) \in \mathbb{R}^{n}: \frac{1}{2\left|a_{0}\right|} \leq\left|\xi_{0}\right|<\frac{1}{2}, 0<\right.$ $\xi_{i} / \xi_{i-1}<1$, for $\left.1 \leq i \leq \ell\right\}$, is a PF MRA $A B$ wavelet. Observe that, unlike the cases (i) and (ii), here the matrices $B$ do not form a group.

In order to generalize Examples 3 and 4 to dimensions $n>2$, we can proceed as follows. Again, let $n=k+\ell$ and $A=\left\{a^{i}: i \in \mathbb{Z}\right\}$, where

$$
a=\left(\left(\begin{array}{ccc}
a_{1} & \cdots & 0 \\
& \ddots & \\
0 & \cdots & a_{k}
\end{array}\right) \quad 0 \quad I_{\ell}\right) \in G L_{n}(\mathbb{R})
$$

and $\left|a_{i}\right|>1$, for each $i=1, \ldots, k$. The matrices $B$ can be chosen according to one of the following patterns. 
(i) Let $k=1, \ell=n-1$ and $B=\left\{b_{j}=\left(\begin{array}{ll}1 & j \\ 0 & I_{\ell}\end{array}\right): j=\left(j_{1}, \ldots, j_{\ell}\right) \in \mathbb{Z}^{\ell}\right\}$. Let $\psi_{1} \in L^{2}(\mathbb{R})$ be a (one-dimensional) band-limited wavelet with respect to the dilation $a_{1}$, having supp $\hat{\psi}_{1} \subset[-\Omega, \Omega], \Omega>0$, and let $\psi_{2}, \ldots, \psi_{n} \in L^{2}(\mathbb{R})$ be also band-limited functions with $\operatorname{supp} \hat{\psi}_{i} \subset[-1,1], i=2, \ldots, n$, and satisfying

$$
\sum_{j \in \mathbb{Z}}\left|\hat{\psi}_{i}(\xi+j)\right|^{2}=1 \quad \text { for a.e. } \xi \in \mathbb{R} \text {, for any } i=2, \ldots, n \text {. }
$$

Now, for any $\omega=\left(\omega_{1}, \ldots, \omega_{n}\right) \in \mathbb{R}^{n}, \omega_{1} \neq 0$, define $\psi \in L^{2}\left(\mathbb{R}^{n}\right)$ by

$$
\hat{\psi}(\omega)=\hat{\psi}_{1}\left(a_{1}^{s} \omega_{1}\right) \hat{\psi}_{2}\left(\frac{\omega_{2}}{\omega_{1}}\right) \times \cdots \times \hat{\psi}_{n}\left(\frac{\omega_{n}}{\omega_{1}}\right),
$$

where $s \in \mathbb{Z}$ satisfies $a_{1}^{s} \geq 2 \Omega$. Then one can show that $\psi$ is a PF $A B$ wavelet.

(ii) Let $n=2 k$ and

$$
B=\left\{b_{j}=\left(I_{k}\left(\begin{array}{ccc}
j_{1} & \ldots & 0 \\
& \ddots & \\
0 & \ldots & j_{k}
\end{array}\right)\right): j=\left(j_{1}, \ldots, j_{k}\right) \in \mathbb{Z}^{k}\right\} .
$$

Let $\psi_{1}, \ldots, \psi_{k} \in L^{2}(\mathbb{R})$ be band-limited wavelets with respect to the dilations $a_{1}, \ldots, a_{k}$, having $\operatorname{supp} \hat{\psi}_{i} \subset\left[-\Omega_{i}, \Omega_{i}\right], \Omega_{i}>0$, and let $\psi_{k+1}, \ldots, \psi_{n} \in$ $L^{2}(\mathbb{R})$ be also band-limited functions with $\operatorname{supp} \hat{\psi}_{i} \subset[-1,1], i=k+$ $1, \ldots, n$, and satisfying (3.3) for any $i=k+1, \ldots, n$. Now, for any $\omega=\left(\omega_{1}, \ldots, \omega_{n}\right) \in \mathbb{R}^{n}, \omega_{1}, \ldots, \omega_{k} \neq 0$, define $\psi \in L^{2}\left(\mathbb{R}^{n}\right)$ by

$\hat{\psi}(\omega)=\hat{\psi}_{1}\left(a_{1}^{s_{1}} \omega_{1}\right) \times \cdots \times \hat{\psi}_{k}\left(a_{k}^{s_{k}} \omega_{k}\right) \hat{\psi}_{k+1}\left(\frac{\omega_{k+1}}{\omega_{1}}\right) \times \cdots \times \hat{\psi}_{n}\left(\frac{\omega_{2 k}}{\omega_{k}}\right)$,

where $s_{k} \in \mathbb{Z}$ satisfies $a_{k}^{s_{k}} \geq 2 \Omega_{k}$. Then one can show that $\psi$ is a $\mathrm{PF}$ $A B$-wavelet.

3.6. Composite wavelet sets. In this section, we consider $A B$-wavelets where $\psi \in L^{2}\left(\mathbb{R}^{n}\right)$ is given by $\hat{\psi}=\chi_{S}$, with $S \subset \widehat{\mathbb{R}}^{n}$. These systems are the analog of the so-called MSF wavelets in the classical wavelet theory.

As a specific example of such systems, consider, for simplicity, the case $n=2$. Let $B=\left\{b_{j}=\left(\begin{array}{ll}1 & j \\ 0 & 1\end{array}\right): j \in \mathbb{Z}\right\}$, and $A=\left\{a^{i}: i \in \mathbb{Z}\right\}$, where $a=\left(\begin{array}{cc}a_{1} & 0 \\ 0 & a_{2}\end{array}\right)$, with $\left|a_{1}\right|>1$ and $a_{2} \neq 0$. Given any $0<\delta \leq 1$, let $S=\left\{\left(\xi_{1}, \xi_{2}\right) \in \mathbb{R}^{2}: \delta \leq\left|\xi_{1}\right| \leq\right.$ $\delta\left|a_{1}\right|$, and $\left.0 \leq \xi_{2} \leq\left|\xi_{1}\right|\right\}$, and define $\psi$ by $\hat{\psi}=\chi_{S}$. A direct computation shows that

$$
\Omega=\bigcup_{i, j \in \mathbb{Z}} S b^{j} a^{i}=\left\{\left(\xi_{1}, \xi_{2}\right) \in \mathbb{R}^{2}: \xi \neq 0\right\},
$$

where the union is disjoint, and this implies that $\psi$ is a $\mathrm{PF} A B$-wavelet.

If, in addition, we take $a \in G L_{n}(\mathbb{Z})$, then, by choosing $S$ appropriately, we can construct systems which are not only PF but even ON bases for $L^{2}\left(\mathbb{R}^{n}\right)$. For example, let $T(0, \alpha)$ be as in Example 1, and let $T(0, \alpha)+(\beta, \gamma)$ denote the triangle obtained by translating $T(0, \alpha)$ by $(\beta, \gamma) \in \mathbb{R}^{2}$. Let $a=\left(\begin{array}{cc}2 & 0 \\ 0 & 2\end{array}\right), B$ as above, and $\psi$ 


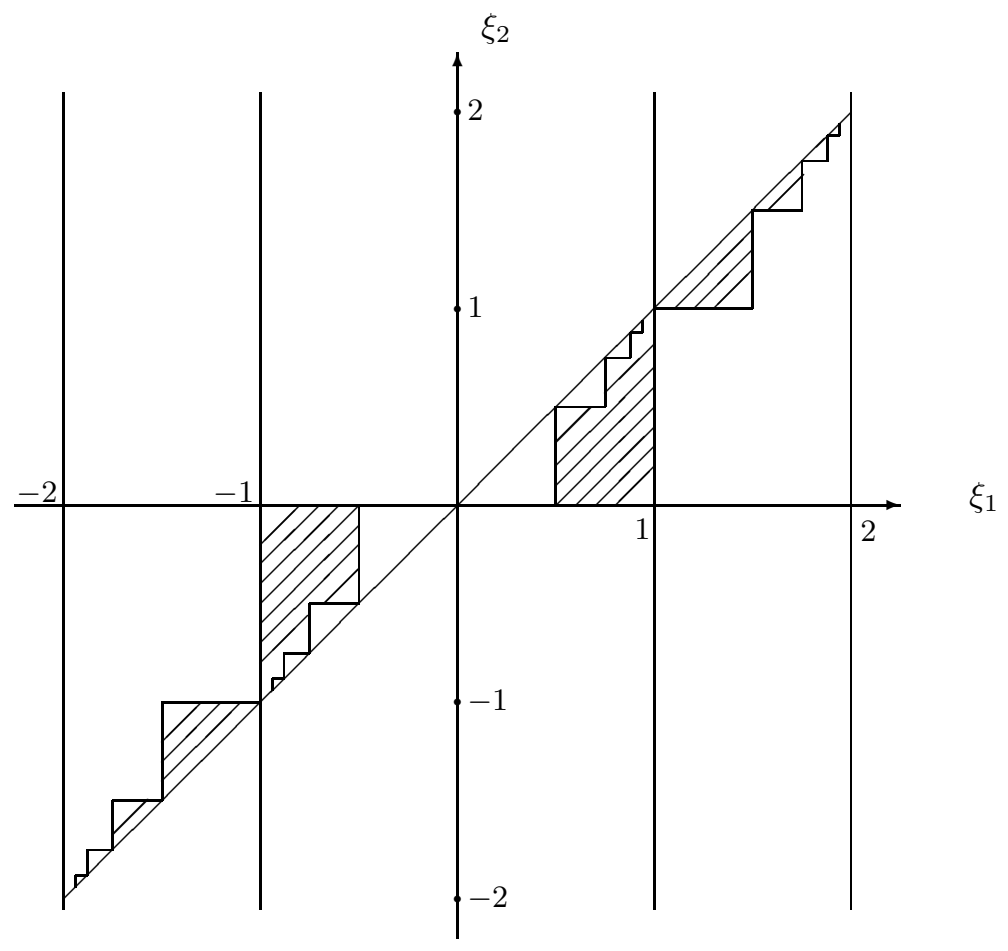

FIgURE 2. A singly generated ON $A B$-wavelet (Section 3.6).

be given by $\hat{\psi}=\chi_{S}$, where $S=S^{+} \cup S^{-}, S^{-}=-S^{+}$and $S^{+}$is the subset of $\mathbb{R}^{2}$ equal to

$$
\left[T(0,1) \backslash \bigcup_{k=1}^{\infty}\left\{T\left(0,2^{-k}\right)+\left(1-2^{-k+1}\right)(1,1)\right\}\right] \cup\left[\bigcup_{k=1}^{\infty}\left\{T\left(0,2^{-k}\right)+\left(2-2^{-k+1}\right)(1,1)\right\}\right] .
$$

This construction is illustrated in Figure 2. It is easy to see that $S$ is a fundamental domain for the $\mathbb{Z}^{2}$-translations and equation (3.4) is satisfied. This shows that $\psi$ is an $\mathrm{ON} A B$-wavelet.

Observe that, since this orthonormal system is generated by a single function, it follows from Theorem 2.2 that $\psi$ is not an ON MRA $A B$-wavelet.

For $a=\left(\begin{array}{ll}2 & 0 \\ 0 & 1\end{array}\right)$, one can construct an analogous unbounded set $S$. Again, $\psi$, where $\hat{\psi}=\chi_{S}$, is not an ON MRA $A B$-wavelet by Theorem 2.2. Higher-dimensional generalizations of these examples can also be obtained.

\section{REFERENCES}

1. E.J. Candès and D.L. Donoho, Ridgelets: a key to higher-dimensional intermittency?, Phil. Trans. Royal Soc. London A 357 (1999), 2495-2509. MR 1721227 (2000g:42047)

2. E.J. Candès and D.L. Donoho, New tight frames of curvelets and optimal representations of objects with $C^{2}$ singularities, Comm. Pure Appl. Math. 57 (2004), 219-266. MR 2012649

3. R.R. Coifman, and F.G. Meyer, Brushlets: a tool for directional image analysis and image compression, Appl. Comp. Harmonic Anal. 5 (1997), 147-187. MR 1448220 (99c:42069)

4. M.N. Do and M. Vetterli, Contourlets, in: Beyond Wavelets, G.V. Welland (ed.), 2003.

5. D.L. Donoho and X. Huo, Beamlets and multiscale image analysis, Lecture notes in computational science and engineering, Springer, 2002. MR 1928566 (2003m:94009) 
6. K. Guo, D. Labate, W. Lim, G. Weiss, and E. Wilson, The theory of wavelets with composite dilations, preprint 2004.

7. E. Hernández, D. Labate, and G. Weiss, A unified characterization of reproducing systems generated by a finite family, II, J. Geom. Anal. 12(4) (2002), 615-662. MR 1916862 (2003j:42036)

8. E. Hernández and G. Weiss, A First Course on Wavelets, CRC Press, Boca Raton, FL, 1996. MR 1408902 (97i:42015)

9. L. Hörmander, The analysis of linear partial differential operators. I. Distribution theory and Fourier analysis. Springer-Verlag, Berlin, 2003. MR 1996773

10. E.M. Stein and G. Weiss, Introduction to Fourier Analysis on Euclidean Spaces, Princeton University Press, Princeton, NJ, 1970. MR 0304972 (46:4102)

11. G.V. Welland (ed.), Beyond Wavelets, Academic Press, San Diego, CA, 2003.

12. G. Weiss, and E. Wilson, The mathematical theory of wavelets, Proceedings of the NATO-ASI Meeting. Harmonic Analysis 2000-A Celebration. Kluwer, 2001. MR 1858791 (2002h:42078)

Department of Mathematics, Southwest Missouri State University, Springfield, MisSOURI 65804

E-mail address: kag026f@smsu.edu

Department of Mathematics, North Carolina State University, Raleigh, North CarOLINA 27695

E-mail address: dlabate@math.ncsu.edu

Department of Mathematics, Washington University, St. Louis, Missouri 63130

E-mail address: wangQ@math.wustl.edu

Department of Mathematics, Washington University, St. Louis, Missouri 63130

E-mail address: guido@math. wustl.edu

Department of Mathematics, Washington University, St. Louis, Missouri 63130

E-mail address: enwilson@math.wustl.edu 